\title{
Impaired Mitochondrial Respiration in Large Cerebral Arteries of Rats with Type 2 Diabetes
}

\author{
Ivan Merdzo Ibolya Rutkai Venkata N.L.R. Sure Catherine A. McNulty \\ Prasad V.G. Katakam David W. Busija \\ Department of Pharmacology, Tulane University School of Medicine, New Orleans, LA, USA
}

\section{Keywords}

Type 2 diabetes - Mitochondria - Mitochondrial respiration .

Cerebral arteries $\cdot$ Cerebral microvessels

\begin{abstract}
Mitochondrial dysfunction has been suggested as a potential underlying cause of pathological conditions associated with type 2 diabetes (T2DM). We have previously shown that mitochondrial respiration and mitochondrial protein levels were similar in the large cerebral arteries of insulin-resistant Zucker obese rats and their lean controls. In this study, we extend our investigations into the mitochondrial dynamics of the cerebral vasculature of 14-week-old Zucker diabetic fatty obese (ZDFO) rats with early T2DM. Body weight and blood glucose levels were significantly higher in the ZDFO group, and basal mitochondrial respiration and proton leak were significantly decreased in the large cerebral arteries of the ZDFO rats compared with the lean controls (ZDFL). The expression of the mitochondrial proteins total manganese superoxide dismutase (MnSOD) and voltage-dependent anion channel (VDAC) were significantly lower in the cerebral microvessels, and acetylated MnSOD levels were significantly reduced in the large arteries of the ZDFO group. Additionally, superoxide production was significantly increased in
\end{abstract}

\section{KARGER}

() 2017 S. Karger AG, Basel

E-Mail karger@karger.com

www.karger.com/jvr the microvessels of the ZDFO group. Despite evidence of increased oxidative stress in ZDFO, exogenous SOD was not able to restore mitochondrial respiration in the ZDFO rats. Our results show, for the first time, that mitochondrial respiration and protein levels are compromised during the early stages of T2DM.

(c) 2017 S. Karger AG, Basel

\section{Introduction}

Diabetes is a global health problem, and type 2 diabetes mellitus (T2DM) accounts for over $90 \%$ of cases. Approximately $9.3 \%$ of people in the USA have diabetes, and the prevalence is increasing dramatically [1]. Although diagnostic and therapeutic possibilities have advanced, diabetes is still a leading cause of renal failure, ischemic heart disease, blindness due to retinopathy, and limb amputations [2-4]. Insulin resistance is the key factor in the development of T2DM [5], and the role of mitochondria in the pathogenesis of insulin resistance and T2DM has been a topic of many studies on different tissues and cells [6]. Some studies have suggested mitochondrial dysfunction as a potential underlying cause of insulin resistance $[7,8]$ whereas others 
have reported a normal mitochondrial respiratory capacity $[9,10]$ and content $[9,11]$ in insulin-resistant humans. A significant number of studies correlates impaired mitochondrial function with T2DM [6, 12-15], but it is not known whether the mitochondrial dysfunction leads to the insulin resistance and the T2DM or vice versa. Our laboratory has previously shown that mitochondria-dependent vasodilatory responses are diminished in the major cerebral arteries of the insulin-resistant Zucker obese rat [16-18] and that the cerebrovascular dysfunction of this rat is mediated by oxidative stress [19]. We have also shown that local cortical microcirculatory responses to different blood flow stimulators were not altered in insulin-resistant Zucker obese rats compared with their lean controls [20]. Recently, we have shown that mitochondrial respiration as well as mitochondrial protein expression levels in the cerebral arteries and microvessels of Zucker obese rats are similar to the controls [21]. These results were obtained for animals in the early stages of insulin resistance, so we have extended these studies to investigate changes in mitochondrial energetics with the development of T2DM. We used Zucker diabetic fatty obese (ZDFO) rats as a model of T2DM and Zucker diabetic fatty lean (ZDFL) rats as the control. We hypothesized that mitochondrial respiration and the expression of mitochondrial proteins would be decreased in the cerebral vasculature of $\mathrm{ZDFO}$ rats. We examined mitochondrial dynamics in the large cerebral arteries and cerebral microvessels from the parenchyma because disease states can influence various cerebral vascular segments differently. Therefore, we used freshly isolated large cerebral arteries to measure mitochondrial respiration, and determined the levels of mitochondrial proteins and superoxide in the isolated cerebral microvessels.

\section{Materials and Methods}

Animal use protocols were approved by the Institutional Animal Care and Use Committee of the Tulane University School of Medicine and complied with the National Institutes of Health guidelines. Animal care was provided by the Department of Comparative Medicine. We used 14-week-old, male ZDFO and ZDFL rats from Charles River Laboratories (Wilmington, MA, USA). Forty animals from each group were used.

\section{ZDFO Model}

The male ZDFO rat maintained on a Purina 5008 diet exhibits obesity, insulin resistance, hyperinsulinemia, hyperglycemia, and T2DM beginning at 6-7 weeks of age. Glucose levels steadily increase until the age of 10-12 weeks, and are then maintained at an average of approximately $27.8 \mathrm{mmol} / \mathrm{L}$ [22].

\section{Blood Glucose Measurements}

Blood samples were taken from the aorta while the animals were deeply anesthetized. Blood glucose levels were measured using a glucometer (Contour Next EZ glucometer, Bayer, Germany) and expressed in $\mathrm{mmol} / \mathrm{L}$ as mean \pm SEM.

\section{Isolation of Cerebral Arteries and Microvessels}

Animals were decapitated after deep anesthesia with 5\% isoflurane (VetOne, Boise, ID, USA). Their brains were removed and placed in ice-cold PBS. All procedures were done on ice or at $4^{\circ} \mathrm{C}$ as described previously [21]. Briefly, large cerebral arteries (anterior and middle cerebral and basilar arteries) were isolated, cleaned, and used for mitochondrial respiration measurements. Cerebral microvessels were then isolated. The cortex was homogenized, centrifuged at $1,000 \mathrm{~g}$ for $10 \mathrm{~min}$; the supernatant was discarded and the pellet was resuspended in $17.5 \%$ dextran $(64-76 \mathrm{kDa}$, Sigma-Aldrich, St. Louis, MO, USA), passed through a 300- $\mu \mathrm{m}$ mesh, and centrifuged at $4,400 \mathrm{~g}$ for $15 \mathrm{~min}$. The same steps were repeated with the resulting supernatant to increase the yield of microvessels. Finally, the microvessel pellets from the previous 2 centrifugations were resuspended together in $17.5 \%$ dextran, centrifuged, and the resulting pellet passed through a $70-\mu \mathrm{m}$ nylon mesh. Captured microvessels were washed with PBS, collected, and used for Western blotting. This microvessel isolation method was validated via immunohistochemistry [21].

\section{Mitochondrial Respiration Measurement}

We used an extracellular flux (XF) analyzer, the Seahorse Bioscience XFe24 analyzer (Seahorse Bioscience, Billerica, MA, USA, currently Agilent Technologies) to measure mitochondrial oxygen consumption rate (OCR), an indicator of mitochondrial respiration [23-25]. To establish the validity and reproducibility of our methodology, we performed separate determinations of mitochondrial respiration on different groups of rats in experiments performed approximately 2 months apart. We used freshly isolated large cerebral arteries for these measurements as described previously $[21,26]$. Briefly, we placed the arteries into the wells of the XF24 islet capture microplate (No. 101122-100, Seahorse Bioscience). The wells were then filled with the XF assay medium (No. 102365-100, Seahorse Bioscience) containing $5.0 \mathrm{~mm} / \mathrm{L}$ glucose and $2 \mathrm{~mm} / \mathrm{L}$ pyruvate and the plates were kept in a non- $\mathrm{CO}_{2}$ incubator at $37^{\circ} \mathrm{C}$ for $20 \mathrm{~min}$. Afterward, the plates were placed into a Seahorse Bioscience XF analyzer, where oxygen and hydrogen ion-sensitive fluorophores repeatedly allowed determination of the oxygen and hydrogen concentrations in the medium surrounding the arteries, thus calculating the OCR. The assay protocol consisted of 8 cycles of baseline measurements, followed by 5 cycles for each treatment. After baseline measurements, the plates were sequentially exposed to $2 \mu \mathrm{M} / \mathrm{L}$ oligomycin, $1 \mu \mathrm{M} / \mathrm{L}$ carbonyl cyanide 4-(trifluoromethoxy) phenylhydrazone (FCCP), and 1.5 $\mu \mathrm{M} / \mathrm{L}$ antimycin plus $1.5 \mu \mathrm{M} / \mathrm{L}$ rotenone to determine the levels of different components of mitochondrial respiration (Fig. 1). Two sample groups were analyzed: ZDFO and ZDFL rats. Protein concentration, (Pierce BCA protein assay kit, Thermo Scientific, Rockford, IL, USA), was used to normalize OCR data, and values were expressed in $\mathrm{pmol} / \mathrm{min} / \mu \mathrm{g}$. After normalization, a series of calculations was performed using OCR data to determine various components of mitochondrial (basal respiration, proton leak, maximal respiration, and spare respiratory capacity) and nonmitochondrial respiration. The same approach was used in the pres- 


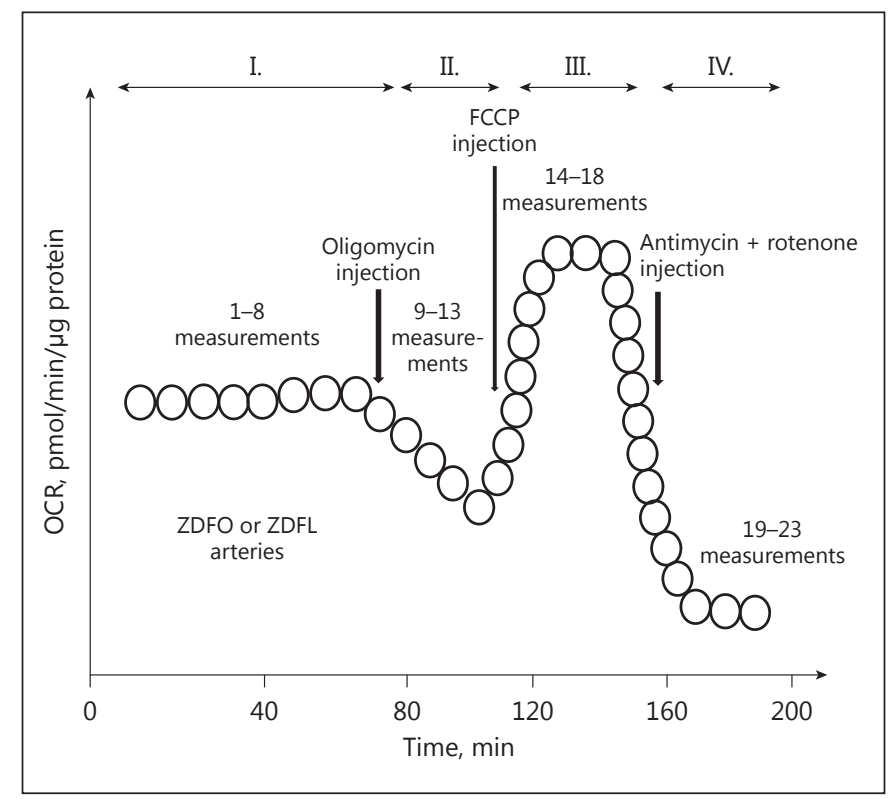

Fig. 1. Schematic illustration of the Seahorse experiment protocol. Roman numerals show different measurement cycles. Time points of different drug administrations are marked with vertical arrows, and the resulting changes in OCR are shown.

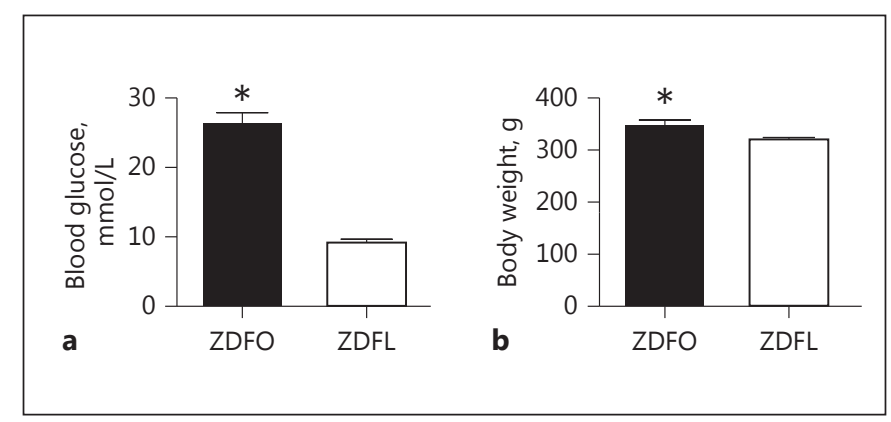

Fig. 2. Blood glucose levels and weight of ZDFL and ZDFO rats. a Blood glucose levels, assessed by the random blood glucose test, were significantly higher in the ZDFO group. $n=40,{ }^{*} p<0.05$ (ZDFL vs. ZDFO). b ZDFO rats exhibited significantly greater body weight than the lean controls. $n=40,{ }^{*} p<0.05$ (ZDFL vs. ZDFO).

ence of a superoxide scavenger, polyethylene glycol-superoxide dismutase (PEG-SOD, 200 units/mL) (Creative Enzymes, Shirley, NY, USA).

\section{Electron Spin Resonance Studies}

Electron spin resonance (ESR) spectroscopy was used to determine the levels of superoxide in the cerebral microvessels of the ZDFL and ZDFO rats, as described previously [17, 21]. Briefly, the 1-hydroxy-3-methoxycarbonyl-2,2,5,5-tetramethyl-pyrrolidine $(\mathrm{CMH})$ spin probe was used to measure superoxide pro- duction in the freshly isolated cerebral microvessels. Diethyldithiocarbamate (DETC; $2.5 \mu \mathrm{mol} / \mathrm{L})$ and deferoxamine $(25$ $\mu \mathrm{mol} / \mathrm{L})$ were dissolved under nitrogen gas bubbling in ice-cold modified Krebs-Hepes (KH) buffer. Microvessels were then placed in a microtube containing $200 \mu \mathrm{mol} / \mathrm{L} \mathrm{CMH}$ solution of $\mathrm{KH}$ buffer with deferoxamine and DETC, and the samples were incubated for $60 \mathrm{~min}$ at $37^{\circ} \mathrm{C}$, placed into the barrel of a $1-\mathrm{mL}$ syringe, and frozen in liquid nitrogen. When samples were ready for measurement, the frozen column of microvessels was placed into a finger dewar (Noxygen Science Transfer and Diagnostics $\mathrm{GmbH}$, Elzach, Germany) containing liquid nitrogen. The finger dewar was placed into the measuring cavity of a benchtop X-band EMX series ESR spectrometer (Bruker Biospin GmbH, Karlsruhe, Germany) and ESR spectra were obtained. Time-dependent formation of reactive oxygen species (ROS) was determined using the following ESR settings: center field, $1.99 \mathrm{~g}$; microwave power, $20 \mathrm{~mW}$; modulation amplitude, $2 \mathrm{~g}$; sweep time, $10 \mathrm{~s}$; number of scans, 10 ; field sweep, $60 \mathrm{~g}$. The measurements of amplitude of the peak and through for each ESR spectrum was normalized to the protein concentrations of the microvessel samples and expressed in arbitrary units (AU).

\section{Western Blot Analysis}

Proteins were harvested as described previously [17, 21, 26]. Briefly, isolated large cerebral arteries and microvessels were homogenized in ice-cold NP40 lysis buffer (Invitrogen, Frederick, MD, USA) supplemented with proteinase inhibitor cocktail (cat. No. P8340; Sigma-Aldrich) and phosphatase inhibitor cocktail (cat. No. P2850; Sigma-Aldrich), and then centrifuged and the supernatant was analyzed. Pierce BCA protein assay was used to determine protein concentration. We then separated protein samples using gel electrophoresis on a 4-20\% SDS-PAGE gradient gel. Proteins were transferred onto a PVDF membrane, blocked with casein blocking buffer (No. 92740200, Li-cor, Lincoln, NE, USA), washed, and incubated overnight with primary antibodies in casein blocking buffer at $4{ }^{\circ} \mathrm{C}$. The following primary antibodies for mitochondrial and nonmitochondrial proteins were used: Anti-Complex II Fp subunit I at 1:1,000 dilution (70 kDa; No. 459200, Invitrogen); Anti-Complex III subunit I core at 1:1,000 dilution (53 kDa; No. 459140, Invitrogen); ATP synthase Complex V subunit $\alpha$ at 1:500 dilution $(50 \mathrm{kDa}$; No. 459240, Invitrogen); anti-voltage-dependent anion channel (VDAC) at 1:1,000 dilution to detect the endogenous levels of total VDAC (32 kDa; No. 4866S, Cell Signaling Technology, Danvers, MA, USA); total endothelial NOS (eNOS) at 1:500 dilution (140 kDa; No. 610297, BD Transduction Laboratories, San José, CA, USA) and its Ser ${ }^{1176}$-phosphorylated form (peNOS) at 1:500 dilution (140 kDa; No. 9571, Cell Signaling Technology); total dynamin-related protein-1 (tDRP) at 1:1,000 dilution (No. 611112, BD Transduction Labs.) and its Ser ${ }^{616}$-phosphorylated form (pDRP) at 1:1,000 dilution (No. 3455, Cell Signaling Technology); total anti-manganese SOD (MnSOD) aa114-220 at 1:5,000 (25 kDa; No. 611581, BD Transduction Labs.); and its acetylated form anti-SOD 2 (acetyl K68) (acetylated MnSOD) at 1:500 dilution (24 kDa, No. Ab137037, Abcam, Cambridge, MA, USA). The loading control was $\beta$-actin at $1: 5,000$ dilution (42 $\mathrm{kDa}$; No. A5441, Sigma-Aldrich). After incubation with the primary antibody, the membranes were washed and incubated at room temperature for $90 \mathrm{~min}$ in $1 \%$ BSA-TBST with secondary goat anti-rabbit IgG at 1:2,500 dilution (No. 7074S, Cell Signaling 


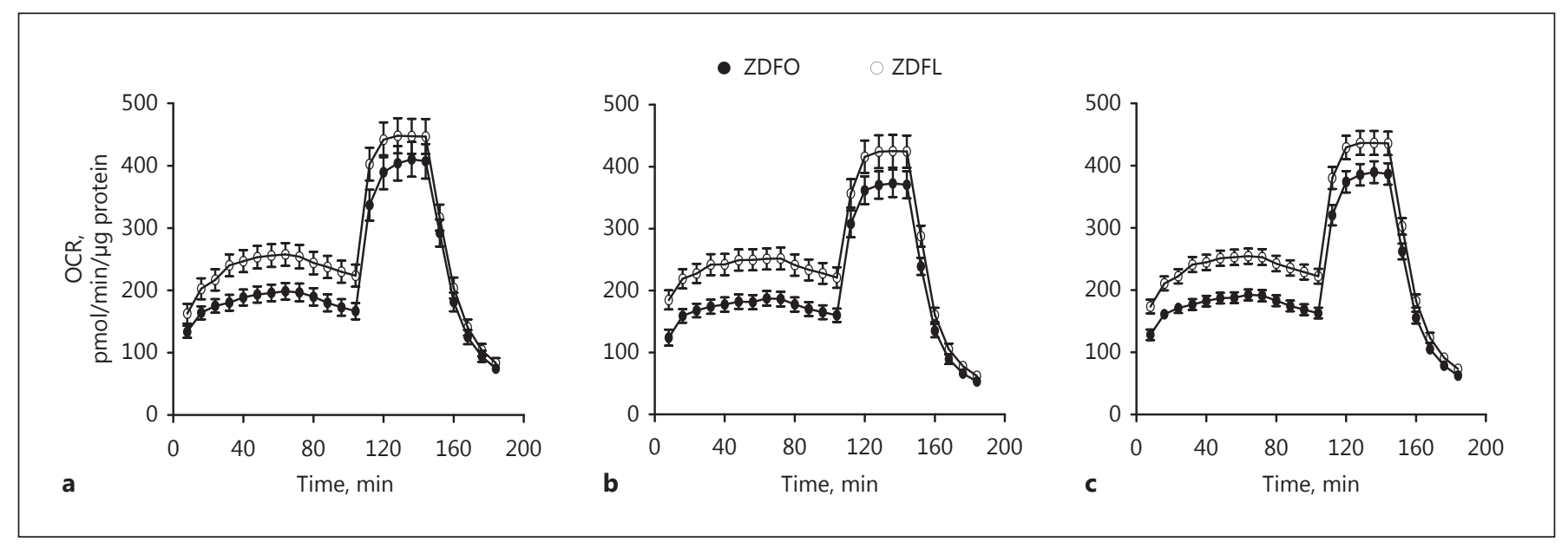

Fig. 3. Mitochondrial respiration profile of large cerebral arteries from ZDFL and ZDFO rats. a First set of experiments. b Second set of experiments. c Merged results from both sets of experiments. The animals represent 2 groups of rats of the same age but in experiments separated by approximately 2 months. Data are expressed as mean \pm SE. $n=23$ per group.

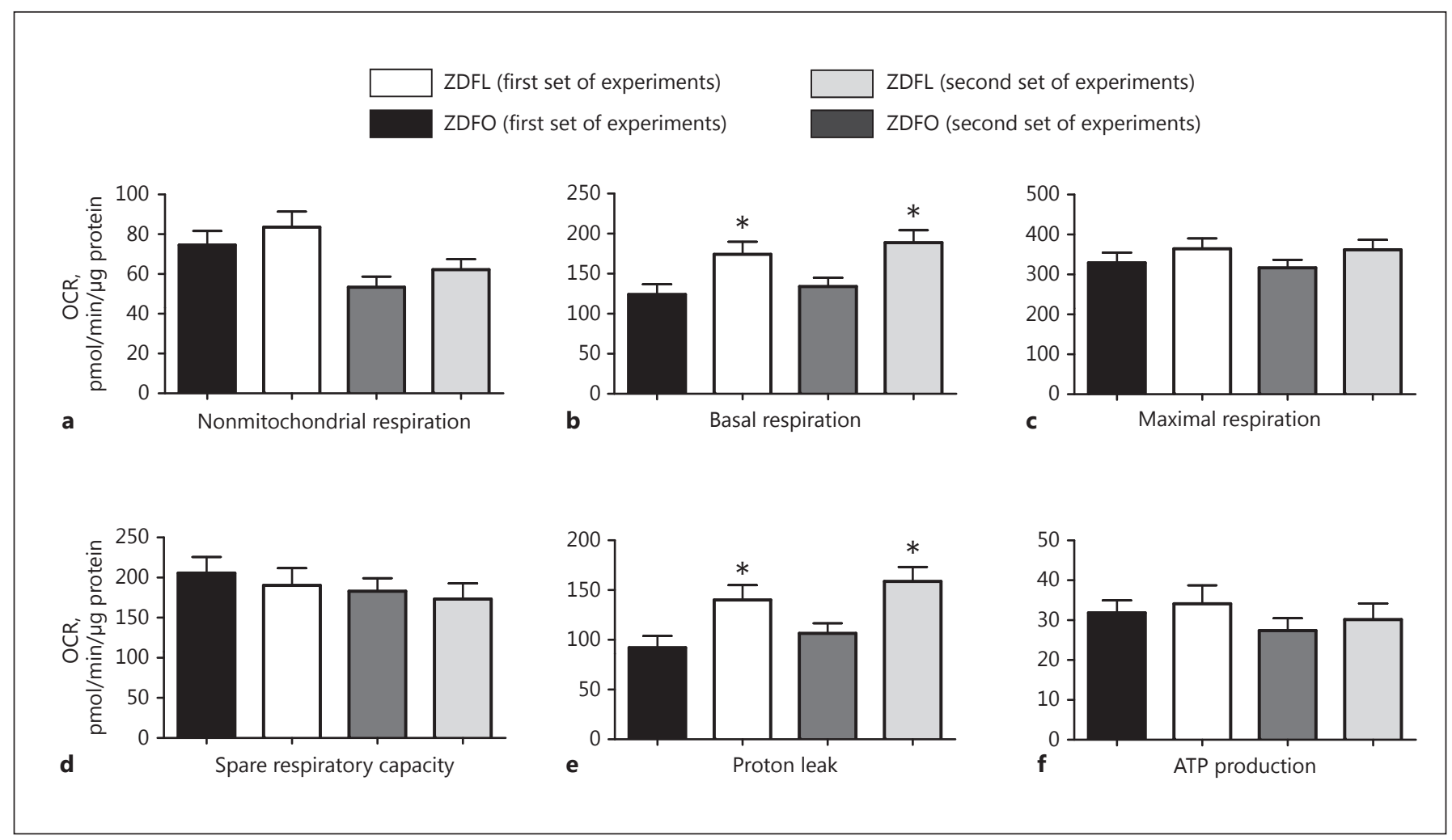

Fig. 4. Mitochondrial respiration components of the large cerebral arteries for 2 individual sets of experiments. Mitochondrial bioenergetic parameters are shown. a Nonmitochondrial respiration. b Basal respiration. c Maximal respiration. $\mathbf{d}$ Spare respiratory capacity. e Proton leak. $\mathbf{f}$ ATP production. Data are expressed as mean \pm SE. $n=29$ per group, ${ }^{*} p<0.05$ (ZDFL vs. ZDFO). 


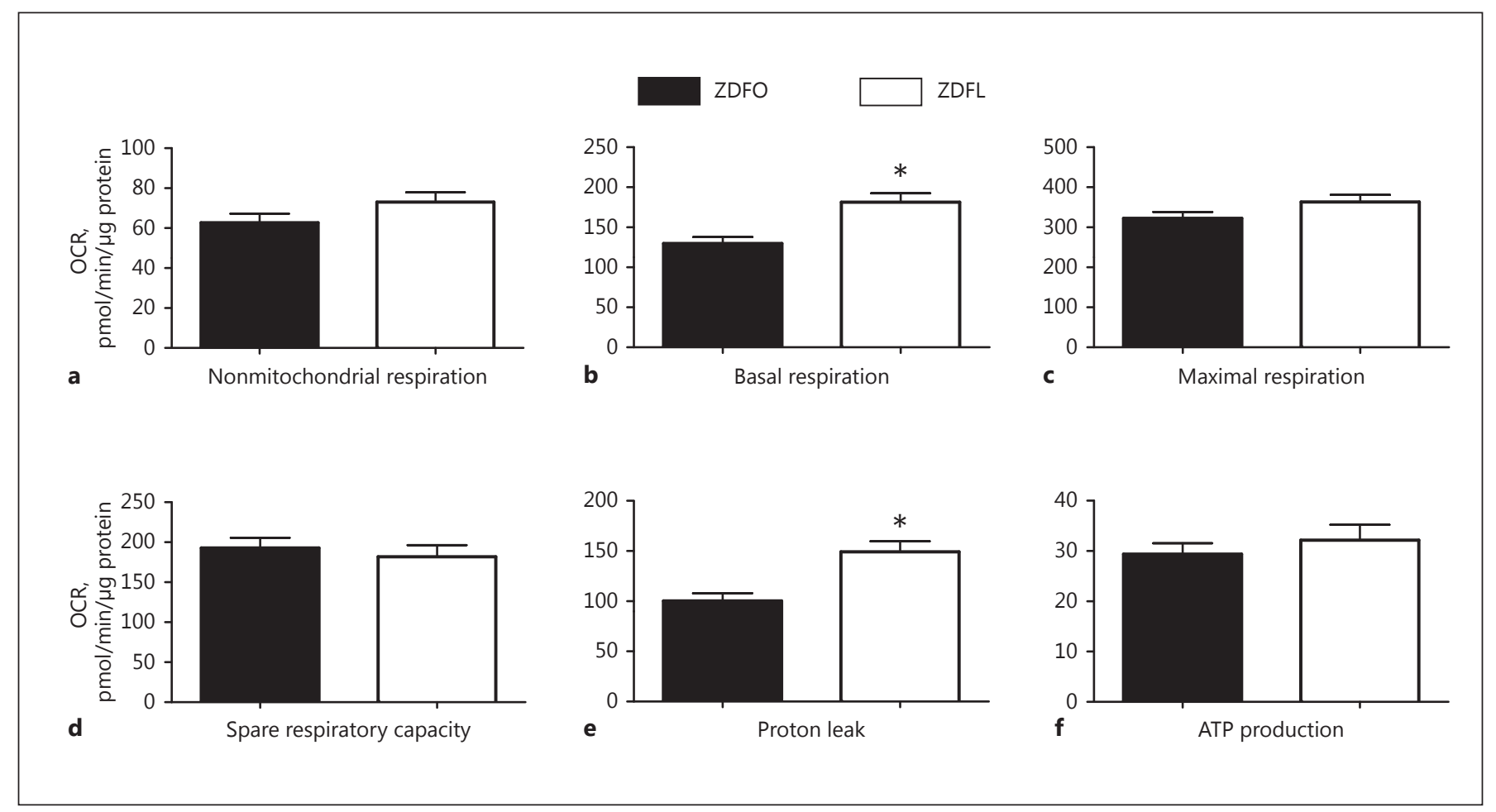

Fig. 5. Mitochondrial respiration components of the large cerebral arteries, merged results. Mitochondrial bioenergetic parameters are shown. a Nonmitochondrial respiration. b Basal respiration. c Maximal respiration. d Spare respiratory capacity. e Proton leak. f ATP production. Data are expressed as mean \pm SE. $n=59$ per group. $* p<0.05$ (ZDFL vs. ZDFO).

Technology) or goat anti-mouse IgG at 1:5,000 dilution (No. 7076P2, Cell Signaling Technology). Immunobands were visualized using chemiluminescence (LumiGLO, Gaithersburg, MD, USA) and autoradiography. Then immunobands were scanned and analyzed using ImageJ software. We quantified the optical density of each band and normalized it to the intensity of the corresponding $\beta$-actin band.

Data Analysis and Statistics

Results were expressed as mean $\pm \mathrm{SE}$; $n$ indicates the number of independent measurements. Data were analyzed by GraphPad Prism software, v5.03 (San Diego, CA, USA) using the unpaired $t$ test. $p<0.05$ was considered statistically significant.

\section{Results}

\section{Animal Weight and Blood Glucose Levels}

The ZDFO group exhibited both significantly higher blood glucose values $(26.33 \pm 1.56$ vs. $9.18 \pm 0.46 \mathrm{mmol} / \mathrm{L}$, $n=40, p<0.05$; Fig. $2 \mathrm{a})$, and body weight $(347.2 \pm 10.18$ vs. $320.4 \pm 3.9$ g, $n=40, p<0.05$; Fig. 2 b) than their lean controls.

Mitochondrial Function in T2DM

Cerebral Arteries

\section{Mitochondrial Respiration Measurements}

Mitochondrial respiration studies were performed twice, separated by approximately 2 months (Fig. 3a, b), on 2 separate groups of 14-week-old rats. Eight independent Seahorse experiments, with 20 animals in each group, confirmed the high level of reproducibility and validated our optimized methods to adopt the Seahorse Bioscience XF analyzer to evaluate the bioenergetics of freshly isolated cerebral arteries. The mitochondrial respiration profile of the large cerebral arteries with the merged results from the first 2 sets of experiments is shown in Figure 3c. Individual components of mitochondrial (basal respiration, ATP production, spare capacity, and proton leak) and nonmitochondrial respiration are shown for both sets of experiments (Fig. 4) as well as for the merged results (Fig. 5). Basal respiration was significantly higher in ZDFL compared with ZDFO (181.4 \pm 10.93 vs. $129.7 \pm 8.211 \mathrm{pmol} / \mathrm{min} / \mu \mathrm{g}$ protein; $n=59, p<$ 0.05 ; Fig. $4 \mathrm{~b}, 5 \mathrm{~b}$ ), and proton leak was also significantly higher in ZDFL compared with ZDFO (149.3 \pm 10.41 vs. $100.3 \pm 7.56 \mathrm{pmol} / \mathrm{min} / \mu \mathrm{g}$ protein; $n=59, p<0.05$; Fig. $4 \mathrm{e}$, 


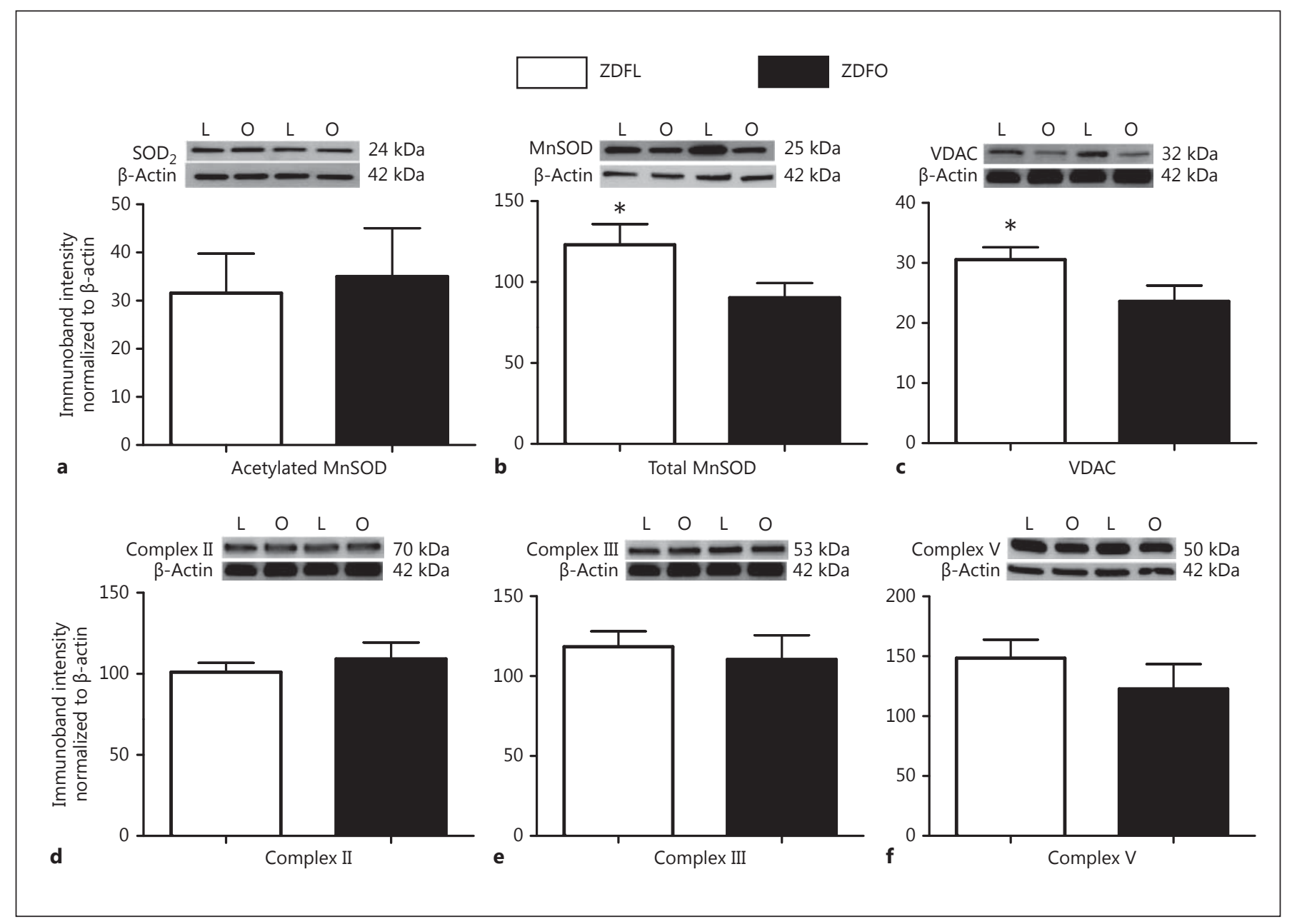

Fig. 6. Mitochondrial protein expression in cerebral microvessels of ZDFL and ZDFO rats. Representative Western blots and summary data. a Acetylated MnSOD, $24 \mathrm{kDa}$. b Total MnSOD, $25 \mathrm{kDa}$. c VDAC, $32 \mathrm{kDa}$. d Complex II, $70 \mathrm{kDa}$. e Complex III, $53 \mathrm{kDa}$. f Complex V, $50 \mathrm{kDA}$. L, lean; O, obese. Data are expressed as mean $\pm \mathrm{SE}$. $n=16$ per group. ${ }^{*} p<0.05$ (ZDFL vs. ZDFO).

5e). Other components of mitochondrial and nonmitochondrial respiration were not significantly different in the 2 groups (Fig. 4, 5).

\section{Protein Expression}

The expression of proteins associated with mitochondrial function (total MnSOD and VDAC) was significantly lower in the microvessels of ZDFO compared with ZDFL (90.35 \pm 9.13 vs. $123.1 \pm 12.77 \%, n=13, p<0.05$; and $23.63 \pm 2.62$ vs. $30.54 \pm 2.07 \%, n=12, p<0.05$; respectively; Fig. 6b, c). Levels of the other mitochondrial and nonmitochondrial proteins (pDRP, tDRP, peNOS, and teNOS; $n=12-16$ per group) were not significantly different between the groups (Fig. 7).
The large cerebral arteries showed higher levels of acetylated MnSOD in ZDFL compared with ZDFO (89.72 \pm 3.17 vs. $52.23 \pm 6.01 \%, n=12, p<0.05$; Fig. 8 a). Levels of the other mitochondrial and nonmitochondrial proteins (pDRP, tDRP, peNOS, and teNOS; $n=12-16$ per group) did not show a significant difference between the groups (Fig. 9).

\section{Superoxide Production in Cerebral Microvessels}

The normalized amplitude of the characteristic ESR signal was significantly higher in the microvessels from ZDFO compared to ZDFL rats $(2,355 \pm 628$ vs. $208 \pm 48 \mathrm{AU}, n=$ $10, p<0.05$; Fig. 10a). Thus, superoxide levels in ZDFO microvessels are 10-fold higher than in ZDFL animals. 


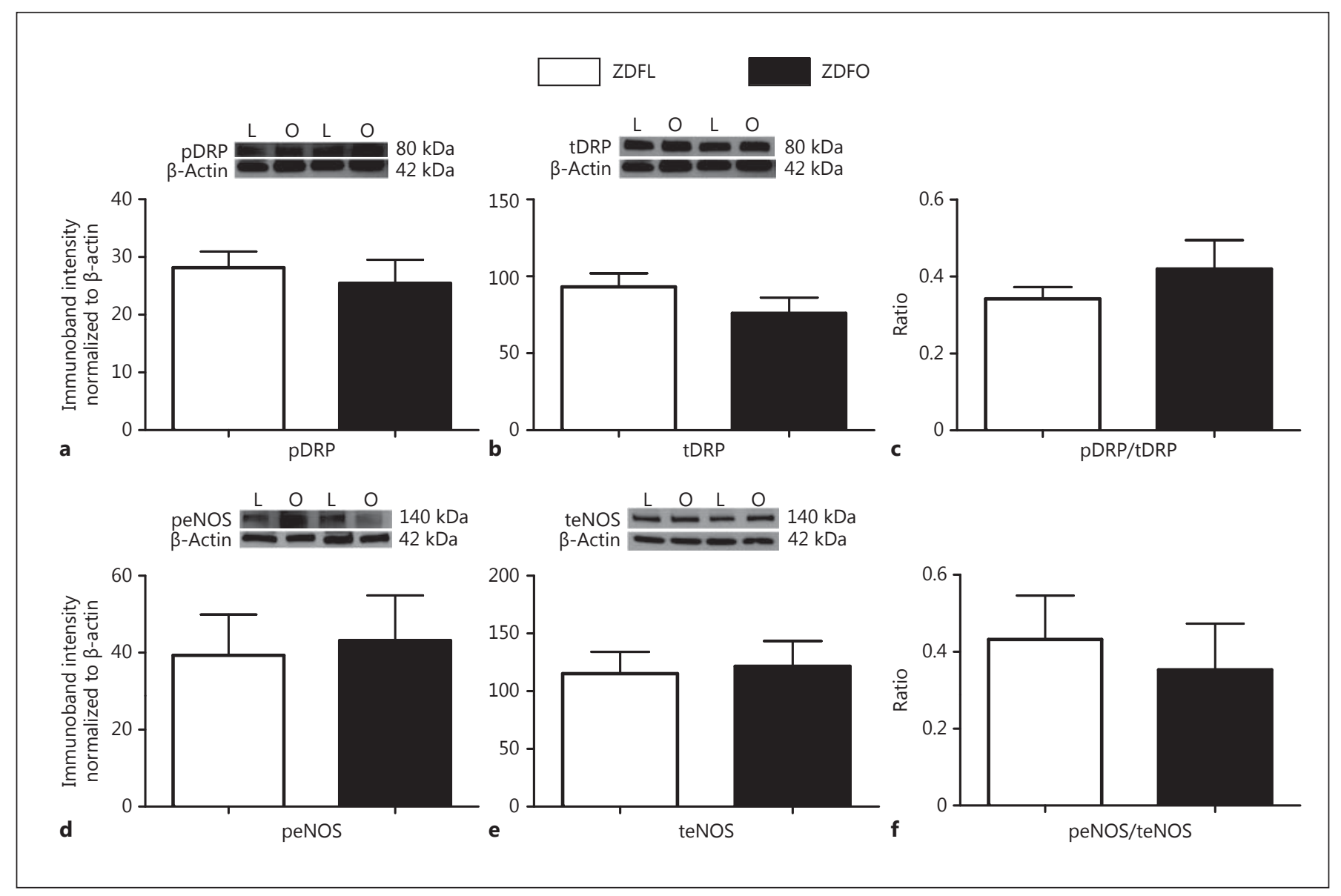

Fig. 7. Mitochondrial and nonmitochondrial protein expression in cerebral microvessels of ZDFL and ZDFO rats. Representative Western blots and summary data. a pDRP, $80 \mathrm{kDa}$. b tDRP, $80 \mathrm{kDa}$. c pDRP/tDRP ratio. d peNOS, $140 \mathrm{kDa}$. e teNOS, $140 \mathrm{kDa}$. f peNOS/teNOS ratio. L, lean; O, obese. Data are expressed as mean $\pm \mathrm{SE}$. $n=16$ per group.

Mitochondrial Respiration Measurements in the Presence of a Superoxide Scavenger

The PEG-SOD treatment did not have a significant effect on mitochondrial respiration and was unable to restore the mitochondrial respiration levels in ZDFO rats (Fig. 10b).

\section{Discussion}

We have directly determined mitochondrial respiration in large cerebral arteries of male ZDFO rats for the first time. Our major new findings include the following: (1) basal mitochondrial respiration is significantly decreased in the large cerebral arteries of ZDFO rats compared to ZDFL rats, (2) proton leak is significantly lower in the large cerebral arteries of ZDFO rats than in ZDFL rats, (3) the expression of mitochondrial proteins (VDAC and total MnSOD) is significantly reduced in the microvessels, and acetylated MnSOD is significantly reduced in the large cerebral arteries of ZDFO rats compared to ZDFL rats. These findings indicate that deterioration of mitochondrial function in the cerebral vasculature starts during early T2DM.

Our laboratory pioneered the use of the Seahorse Bioscience XFe24 analyzer to determine mitochondrial respiration in freshly isolated large cerebral arteries. Due to factors such as intra-animal variation, trauma, and delays during removal of the arteries (and other intangible factors related to any experiment), it was important to examine the reproducibility of our results. In this study, we demonstrated that we could achieve virtually identical 


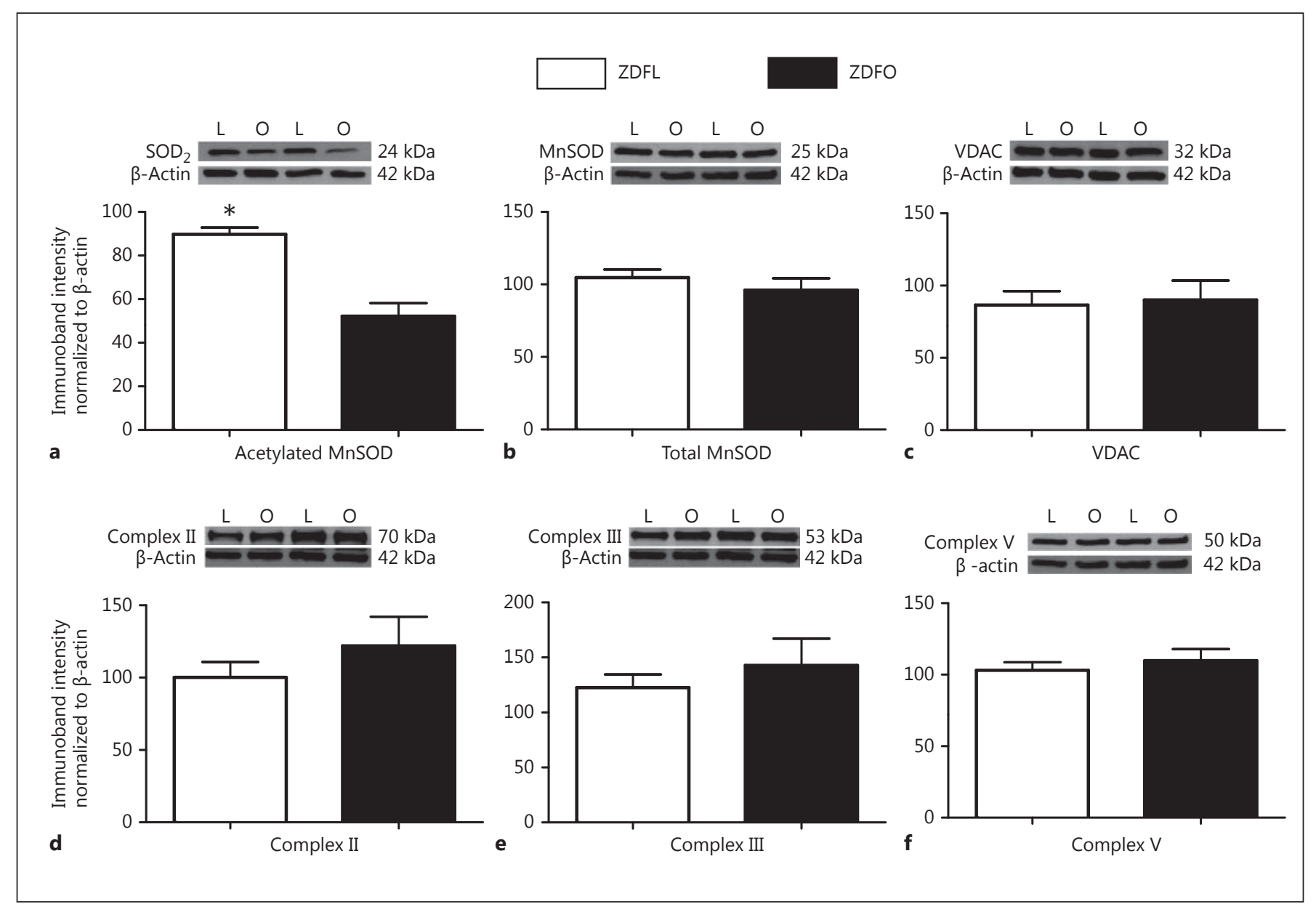

Fig. 8. Mitochondrial protein expression in large cerebral arteries of ZDFL and ZDFO rats. Representative Western blots and summary data. a Acetylated MnSOD, $24 \mathrm{kDa}$. b Total MnSOD, $25 \mathrm{kDa}$. c VDAC, $32 \mathrm{kDa}$. d Complex II, $70 \mathrm{kDa}$. e Complex III, $53 \mathrm{kDa}$. f Complex V, $50 \mathrm{kDa}$. L, lean; O, obese. Data are expressed as mean $\pm \mathrm{SE}$. $n=16$ per group. ${ }^{*} p<0.05$ (ZDFL vs. ZDFO).

measurements of mitochondrial respiration in the large arteries from ZDFO and ZDFL rats of the same age in experiments separated by 2 months. Although not specifically detailed in this paper, we found a similar reproducibility of results with Western blotting.

Although the role of mitochondria in the pathogenesis of insulin resistance and T2DM has been the subject of many studies, additional information is necessary to further elucidate the exact mechanism in these processes. Considering the conflicting reports $[6-11,15]$, the question that remains is whether the impairment of mitochondrial function causes insulin resistance and T2DM or whether it derives from it. It is important to note that mitochondrial function has different implications regarding diabetes in different tissues and cells [6], and that the stud- ies cited were performed on different samples and at different stages of the disease. Different tissues and cells also exhibit different mitochondrial morphology under certain conditions [27-29]. We have found that even within the same population of vascular cells during insulin resistance, one aspect of mitochondrial function is altered (arterial dilation to mitochondrial depolarizing agents) while other aspects are unchanged (total cerebral artery mitochondrial respiration and protein levels) $[18,19,21]$. One study argues that if mitochondrial impairment leads to T2DM, it should be present in the early stages of insulin resistance, but the investigators found normal mitochondrial respiration and content in these early stages [9].

Previous studies from our lab also focused on the early stages of insulin resistance in the absence of diabetes, 


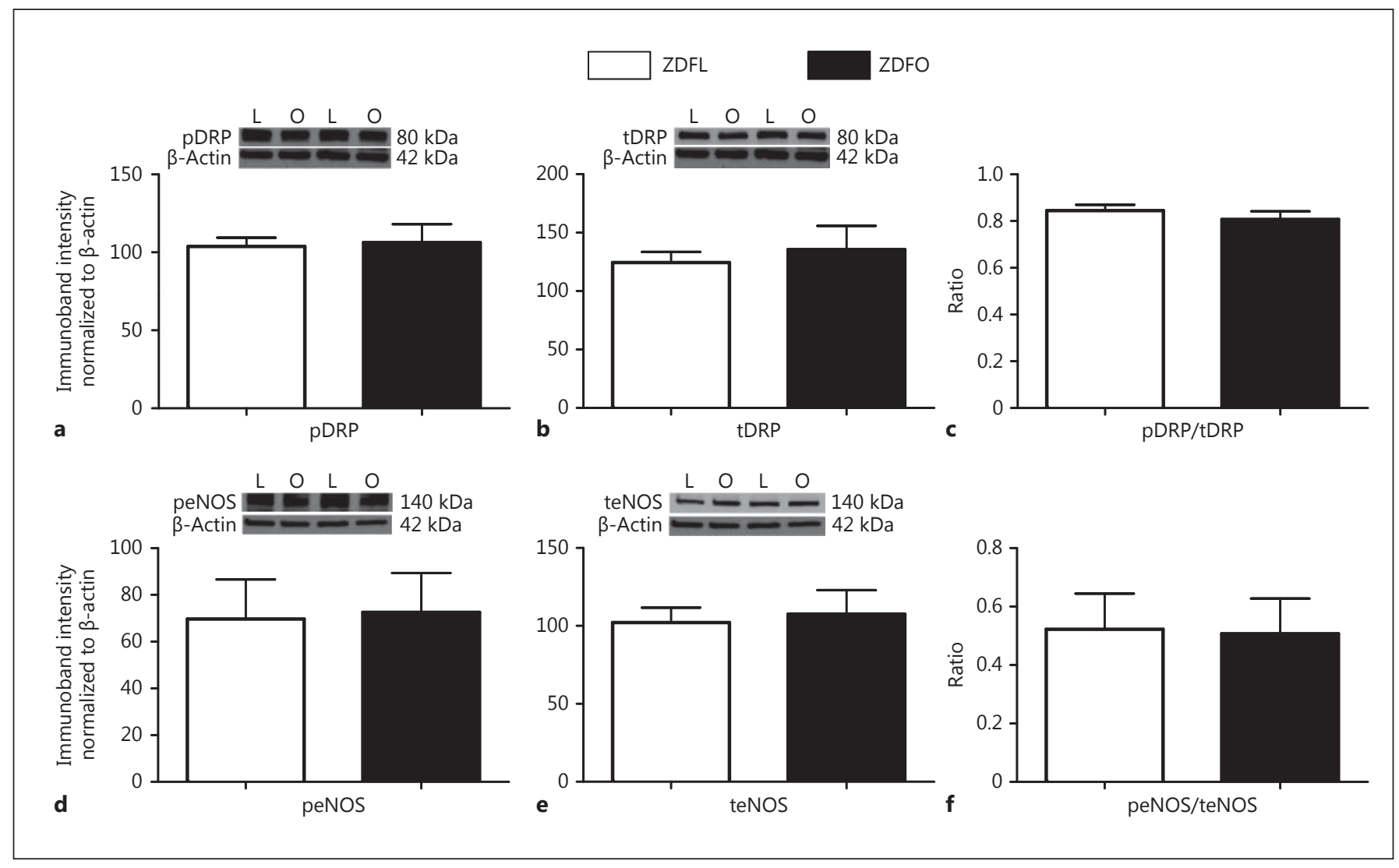

Fig. 9. Mitochondrial and nonmitochondrial protein expression in large cerebral arteries of ZDFL and ZDFO rats. Representative Western blots and summary data. a pDRP, $80 \mathrm{kDa}$. b tDRP, $80 \mathrm{kDa}$. c pDRP/tDRP ratio. d peNOS, $140 \mathrm{kDa}$. e teNOS, $140 \mathrm{kDa}$. f peNOS/teNOS ratio. L, lean; O, obese. Data are expressed as mean $\pm \mathrm{SE}$. $n=16$ per group.

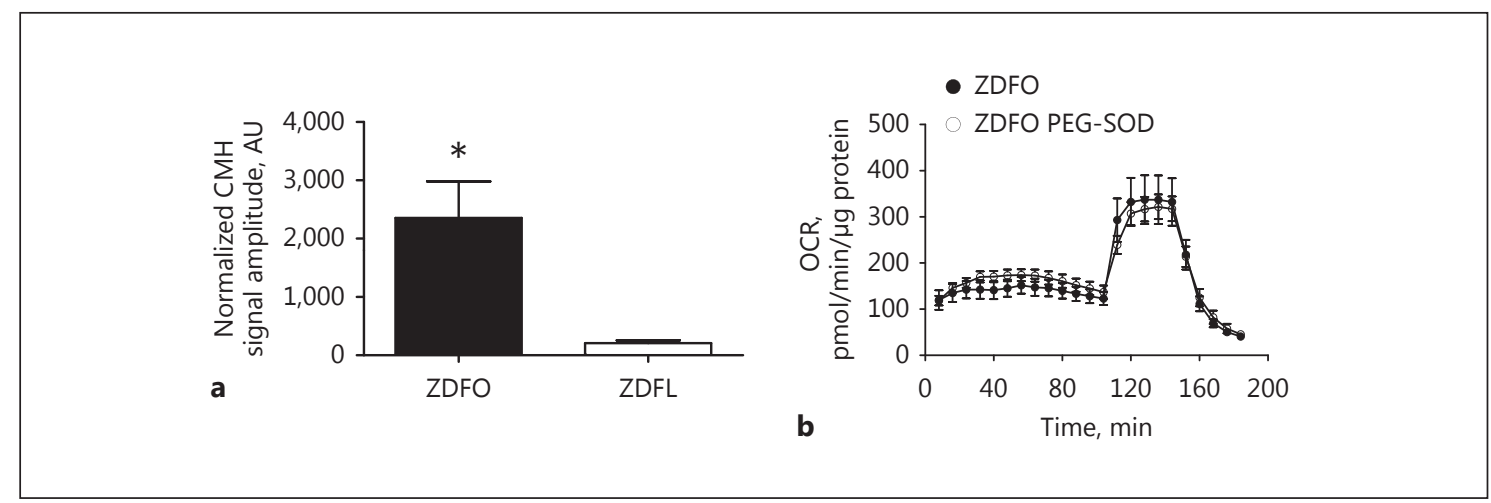

Fig. 10. Superoxide levels in cerebral microvessels of ZDF rats and the effect of PEG-SOD on mitochondrial respiration profile. a Superoxide levels were significantly increased in the microvessels of the ZDFO group compared to the ZDFL group. $n=10,{ }^{*} p<0.05$ (ZDFL vs. ZDFO). $\mathbf{b}$ Treatment with PEG-SOD did not ameliorate mitochondrial respiration in the ZDFO group. 
and found decreased vasodilation of the insulin-resistant rat's cerebral arteries, that was mediated by mitochondrial depolarization $[16,17]$. Excess production of ROS plays a key role in reduced vasodilatory responses in the cerebral arteries of the insulin-resistant rats since pretreatment with SOD restored all of the examined dilator responses $[19,30]$. We have also shown that rosuvastatin improves cerebrovascular function during insulin resistance by inhibiting NAD(P)H oxidase-dependent superoxide production [31]. This finding implies that irreversible changes have not yet occurred in the cerebral vasculature this early in the disease progression. Although functional tests showed lower responses in the cerebral arteries of insulin-resistant rats, microcirculatory responses to different pharmacological and physiological stimuli were similar between the insulin-resistant and control groups [20].

Considering that impaired cerebrovascular responses to mitochondrial depolarizing agents occur during insulin resistance, we conducted further experiments to directly measure mitochondrial respiration in the cerebral arteries of insulin-resistant rats, and found very similar respiration profiles and similar mitochondrial protein expression in the insulin-resistant and control groups [21]. However, we found significantly higher superoxide levels in cerebral microvessels; this is consistent with previous findings in which oxidative stress played a significant role, even early on in the development of insulin resistance and T2DM. It has been reported that high glucose levels during diabetes lead to an increase in ROS production in the cerebral vascular endothelium [32], by creating excess electron donors that generate an increase in mitochondrial membrane potential. A high mitochondrial membrane potential inhibits complex III of the respiratory chain. It also increases the half-life of the free radical intermediate of coenzyme q, necessary for the reduction of oxygen to superoxide. Thus, this sequence of events leads to abnormal ROS levels [33]. Increased levels of free radicals can then target proteins, lipids, polysaccharides, and DNA, causing mitochondrial respiration changes. In this study, we have shown that basal mitochondrial respiration in the cerebral arteries decreases when diabetes is fully developed, which suggests that this is a consequence of T2DM. We have also shown that the cerebral arteries of the ZDFO rats had significantly higher levels of proton leak. Proton leak indicates the flow of the proton gradient across the inner mitochondrial membrane that is not used to drive the ATP synthase. It can be affected by ROS generation, mitochondrial membrane composition [34, 35], and un- coupling proteins [36]. Many important roles of uncoupling proteins have been discussed, including the protection of the cells from oxidative stress damage and mitigation of insulin secretion [37]. Increased proton leak in ZDFO arteries may be a compensatory response to increased ROS generation.

A novel finding was that levels of total MnSOD, located in the matrix, and VDAC, located in the outer membrane, were significantly lower in the cerebral microvessels of the ZDFO rats than in the control group. MnSOD and VDAC are considered key mitochondrial proteins involved in responses to stress and apoptosis. Both are encoded by nuclear DNA and incorporated into the mitochondria after being produced in the cytosol. While the other mitochondrial proteins examined did not differ between groups, the reductions in total MnSOD and VDAC show that structural aspects of the mitochondria are beginning to be adversely affected in parenchymal blood vessels at an early stage of T2DM. Although total levels of total MnSOD in the large surface arteries were similar in both groups, the levels of acetylated MnSOD, which represents a less active form of the enzyme, were reduced in the large arteries of the ZDFO rats. Reversible acetylation is an important regulating mechanism of protection against oxidative stress. The acetylation of mitochondrial proteins plays a role in maintaining and regulating mitochondrial ROS levels. The Sirt3 gene appears to be a crucial element here. Sirt3 serves as main mitochondrial deacetylase and decreases MnSOD acetylation and thereby increases MnSOD activity [38]. These results indicate that the SOD capacity of this enzyme is enhanced in the large arteries of diabetic rats, possibly in response to oxidative stress. These findings of functional, but not structural, changes in the mitochondria of the large cerebral arteries are consistent with studies on the dilator responses of insulin-resistant and diabetic animals, where responsiveness is restored quickly in the presence of ROS scavengers. Although the response mode of MnSOD to diabetes differs in large surface arteries compared with parenchymal microvessels and cerebral vascular segments that are subjected to different environmental stimuli, a common feature is that a major ROS scavenging enzyme is substantially affected by even early T2DM. As we observed in cerebral microvessels, increased superoxide production may represent the initiating event. It has been reported that downregulation of VDAC in coronary endothelial cells of type 1 diabetic mice decreases the generation of mitochondrial superoxide [39]. Our finding of lower VDAC levels in the cerebral microvessels of the ZDFO rats might 
represent another compensatory mechanism, in addition to altered MnSOD dynamics, to minimize the detrimental consequences of increased oxidative stress in T2DM. Further studies are required to elucidate the roles of MnSOD and VDAC and other mitochondrial proteins during disease states.

There were some limitations to our study. Only male rats were used. Future studies on female rats or mice as well as on older rodents with more established T2DM are planned. The mitochondrial respiration studies were only performed on the large arteries because of the insufficient mass of the microvessels and the technical limitations of the Seahorse Bioscience XF analyzer. We are exploring ways to conduct similar investigations on microvessels. Finally, we have not yet been able to differentiate the cell-specific mitochondrial responses using large cerebral arteries and microvessels. In future studies, we will explore the responses of the individual cell types by culturing endothelial and vascular smooth muscle cells.

The complex interactions between insulin resistance, T2DM, and mitochondrial function still require additional studies to elucidate all of the underlying mechanisms. The functional characteristics of the cerebral vasculature seem to be impaired, even in the early stages of insulin resistance. However, decrements in the mitochondrial respiration and certain mitochondrial proteins in the cerebral vessels appear to be a consequence of the disease rather than the underlying cause.

\section{Author Contributions}

I.M. and D.W.B. conceived and designed the experiments; I.M., I.R., V.N.L.R.S., C.A.M., and P.V.G.K. performed experiments; I.M. and D.W.B. analyzed data, interpreted experimental results, and prepared figures; I.M. drafted the manuscript; I.M., I.R., V.N.L.R.S., C.A.M., P.V.G.K., and D.W.B. edited and revised the manuscript and approved the final version of the manuscript.

\section{Acknowledgements}

We thank Nancy Busija, MA, for editorial assistance. We thank Dana Liu for technical help.

\section{Sources of Funding}

This work was supported by National Institutes of Health grants (D.W.B.: HL-077731 and HL093554; P.V.G.K.: NS094834), American Heart Association Greater Southeast Affiliate postdoctoral fellowship grant (I.R.: 15POST23040005), American Heart Association Greater Southeast Affiliate predoctoral fellowship grant (V.N.L.R.S.: 16PRE31450006), American Heart Association National Center Scientist Development Grant (P.V.G.K.: 14SDG20490359), and Louisiana Board of Regents Support Fund-Research Competitiveness Subprogram (P.V.G.K.: LEQSF [2014-17]-RD-A-11). This research was also supported by the Louisiana Board of Regents Endowed Chairs for Eminent Scholars program (D.W.B.).

\section{Disclosure Statement}

The authors have no conflicts of interest to disclose.

\section{References}

1 Centers for Disease Control and Prevention: National Diabetes Statistics Report: Estimates of Diabetes and Its Burden in the United States, 2014. Atlanta, US Department of Health and Human Services, 2014.

2 Deshpande AD, Harris-Hayes M, Schootman M: Epidemiology of diabetes and diabetes-related complications. Phys Ther 2008;88:12541264.

3 Ahola AJ, Saraheimo M, Forsblom C, Hietala K, Sintonen H, Groop PH: Health-related quality of life in patients with type 1 diabetes - association with diabetic complications (the FinnDiane Study). Nephrol Dial Transplant 2010;25:1903-1908.

4 Martin CL, Albers J, Herman WH, Cleary P, Waberski B, Greene DA, Stevens MJ, Feldman EL: Neuropathy among the diabetes control and complications trial cohort 8 years after trial completion. Diabetes Care 2006;29: 340-344.
5 Alberti KG, Zimmet P, Shaw J: Metabolic syndrome - a new world-wide definition. A Consensus Statement from the International Diabetes Federation. Diabetic Med 2006;23:469480.

6 Sivitz WI, Yorek MA: Mitochondrial dysfunction in diabetes: from molecular mechanisms to functional significance and therapeutic opportunities. Antioxid Redox Signal 2010;12:537-577.

7 Kelley DE, He J, Menshikova EV, Ritov VB: Dysfunction of mitochondria in human skeletal muscle in type 2 diabetes. Diabetes 2002; 51:2944-2950.

8 Ritov VB, Menshikova EV, He J, Ferrell RE, Goodpaster BH, Kelley DE: Deficiency of subsarcolemmal mitochondria in obesity and type 2 diabetes. Diabetes 2005;54:8-14.
9 Fisher-Wellman KH, Weber TM, Cathey BL, Brophy PM, Gilliam LA, Kane CL, Maples JM, Gavin TP, Houmard JA, Neufer PD: Mitochondrial respiratory capacity and content are normal in young insulin-resistant obese humans. Diabetes 2014;63:132-141.

10 Lefort N, Glancy B, Bowen B, Willis WT, Bailowitz Z, De Filippis EA, Brophy C, Meyer C, Hojlund K, Yi Z, Mandarino LJ: Increased reactive oxygen species production and lower abundance of complex I subunits and carnitine palmitoyltransferase $1 \mathrm{~B}$ protein despite normal mitochondrial respiration in insulinresistant human skeletal muscle. Diabetes 2010;59:2444-2452.

11 Karakelides H, Irving BA, Short KR, O’Brien P, Nair KS: Age, obesity, and sex effects on insulin sensitivity and skeletal muscle mitochondrial function. Diabetes 2010;59:89-97. 
12 Jeong EM, Chung J, Liu H, Go Y, Gladstein S, Farzaneh-Far A, Lewandowski ED, Dudley SC Jr: Role of mitochondrial oxidative stress in glucose tolerance, insulin resistance, and cardiac diastolic dysfunction. J Am Heart Assoc 2016;5:e003046.

13 Choo HJ, Kim JH, Kwon OB, Lee CS, Mun JY, Han SS, Yoon YS, Yoon G, Choi KM, Ko YG: Mitochondria are impaired in the adipocytes of type 2 diabetic mice. Diabetologia 2006;49: 784-791.

14 Petersen KF, Dufour S, Befroy D, Garcia R, Shulman GI: Impaired mitochondrial activity in the insulin-resistant offspring of patients with type 2 diabetes. N Engl J Med 2004;350: 664-671.

15 Petersen KF, Befroy D, Dufour S, Dziura J, Ariyan C, Rothman DL, DiPietro L, Cline GW, Shulman GI: Mitochondrial dysfunction in the elderly: possible role in insulin resistance. Science 2003;300:1140-1142.

16 Katakam PV, Domoki F, Snipes JA, Busija AR, Jarajapu YP, Busija DW: Impaired mitochondria-dependent vasodilation in cerebral arteries of Zucker obese rats with insulin resistance. Am J Physiol Regul Integr Comp Physiol 2009;296:R289-R298.

17 Katakam PV, Gordon AO, Sure VN, Rutkai I, Busija DW: Diversity of mitochondria-dependent dilator mechanisms in vascular smooth muscle of cerebral arteries from normal and insulin-resistant rats. Am J Physiol Heart Circ Physiol 2014;307:H493-H503.

18 Busija DW, Rutkai I, Dutta S, Katakam PV: Role of mitochondria in cerebral vascular function: energy production, cellular protection, and regulation of vascular tone. Compr Physiol 2016;6:1529-1548.

19 Erdos B, Snipes JA, Miller AW, Busija DW: Cerebrovascular dysfunction in Zucker obese rats is mediated by oxidative stress and protein kinase C. Diabetes 2004;53:1352-1359.

20 Institoris A, Lenti L, Domoki F, Wappler E, Gaspar T, Katakam PV, Bari F, Busija DW: Cerebral microcirculatory responses of insulin-resistant rats are preserved to physiological and pharmacological stimuli. Microcirculation 2012;19:749-756.
21 Merdzo I, Rutkai I, Tokes T, Sure VN, Katakam PV, Busija DW: The mitochondrial function of the cerebral vasculature in insulin resistant Zucker obese rats. Am J Physiol Heart Circ Physiol 2016;310:H830-H838.

22 Shafrir E: Animal Models of Diabetes: Frontiers in Research, ed 2. Boca Raton, CRC Press, 2007.

23 Brand MD, Nicholls DG: Assessing mitochondrial dysfunction in cells. Biochem J 2011;435:297-312.

24 Gerencser AA, Chinopoulos C, Birket MJ, Jastroch M, Vitelli C, Nicholls DG, Brand MD: Quantitative measurement of mitochondrial membrane potential in cultured cells: calcium-induced de- and hyperpolarization of neuronal mitochondria. J Physiol 2012;590: 2845-2871.

25 Hill BG, Benavides GA, Lancaster JR Jr, Ballinger S, Dell'Italia L, Jianhua Z, Darley-Usmar VM: Integration of cellular bioenergetics with mitochondrial quality control and autophagy. Biol Chem 2012;393:1485-1512.

26 Rutkai I, Dutta S, Katakam PV, Busija DW: Dynamics of enhanced mitochondrial respiration in female compared with male rat cerebral arteries. Am J Physiol Heart Circ Physiol 2015;309:H1490-H1500.

27 Chalmers S, Saunter CD, Girkin JM, McCarron JG: Flicker-assisted localization microscopy reveals altered mitochondrial architecture in hypertension. Sci Rep 2015;5:16875.

28 Ryan J, Dasgupta A, Huston J, Chen KH, Archer SL: Mitochondrial dynamics in pulmonary arterial hypertension. J Mol Med 2015; 93:229-242.

29 Marsboom G, Toth PT, Ryan JJ, Hong Z, Wu $\mathrm{X}$, Fang YH, Thenappan T, Piao L, Zhang HJ, Pogoriler J, Chen Y, Morrow E, Weir EK, Rehman J, Archer SL: Dynamin-related protein 1-mediated mitochondrial mitotic fission permits hyperproliferation of vascular smooth muscle cells and offers a novel therapeutic target in pulmonary hypertension. Circ Res 2012;110:1484-1497.

30 Erdos B, Simandle SA, Snipes JA, Miller AW, Busija DW: Potassium channel dysfunction in cerebral arteries of insulin-resistant rats is mediated by reactive oxygen species. Stroke 2004;35:964-969.
31 Erdos B, Snipes JA, Tulbert CD, Katakam P, Miller AW, Busija DW: Rosuvastatin improves cerebrovascular function in Zucker obese rats by inhibiting $\mathrm{NAD}(\mathrm{P}) \mathrm{H}$ oxidasedependent superoxide production. Am J Physiol Heart Circ Physiol 2006;290:H1264H1270.

32 Carvalho C, Katz PS, Dutta S, Katakam PV, Moreira PI, Busija DW: Increased susceptibility to amyloid-beta toxicity in rat brain microvascular endothelial cells under hyperglycemic conditions. J Alzheimer Dis 2014;38:7583.

33 Shah GN, Morofuji Y, Banks WA, Price TO: High glucose-induced mitochondrial respiration and reactive oxygen species in mouse cerebral pericytes is reversed by pharmacological inhibition of mitochondrial carbonic anhydrases: implications for cerebral microvascular disease in diabetes. Biochem Biophys Res Commun 2013;440:354-358.

34 Brand MD, Hafner RP, Brown GC: Control of respiration in non-phosphorylating mitochondria is shared between the proton leak and the respiratory chain. Biochem J 1988; 255:535-539.

35 Rolfe DF, Brown GC: Cellular energy utilization and molecular origin of standard metabolic rate in mammals. Physiol Rev 1997;77: 731-758.

36 Busiello RA, Savarese S, Lombardi A: Mitochondrial uncoupling proteins and energy metabolism. Front Physiol 2015;6:36.

37 Brand MD, Esteves TC: Physiological functions of the mitochondrial uncoupling proteins UCP2 and UCP3. Cell Metab 2005;2: 85-93.

38 Ozden O, Park SH, Kim HS, Jiang H, Coleman MC, Spitz DR, Gius D: Acetylation of MnSOD directs enzymatic activity responding to cellular nutrient status or oxidative stress. Aging 2011;3:102-107.

39 Sasaki K, Donthamsetty R, Heldak M, Cho YE, Scott BT, Makino A: VDAC: old protein with new roles in diabetes. Am J Physiol Cell Physiol 2012;303:C1055-C1060. 